\title{
Erratum to: Dissecting deuteron Compton scattering I: The observables with polarised initial states
}

\author{
Harald W. Grießhammer ${ }^{\mathrm{a}}$ \\ Institute for Nuclear Studies, Department of Physics, George Washington University, Washington, DC 20052, USA \\ Original article: Eur. Phys. J. A (2013) 49: 100, https://doi.org/10.1140/epja/i2013-13100-2 \\ Received: 19 March 2018 \\ Published online: 16 April 2018 - (C) Società Italiana di Fisica / Springer-Verlag 2018
}

In addition to the changes of the first erratum (Eur. Phys. J. A 53, 113 (2017)), the results of eqs. (17) and (18) (target polarisations and double polarisations with circularly polarised beams) must be multiplied by a factor of 2 for $M>0$. Therefore, the results for the corresponding asymmetries and rates in figs. 4, 7 to 10, 12,14 and 15 also increase by a factor of 2 . This mandates the following text changes:

- p. 13, left, replace the third sentence by "With magnitudes of up to 0.7 , the largest asymmetries are $\Sigma^{\text {lin }}, T_{1 M}^{\text {circ }}$ and $T_{22}^{\operatorname{lin}}$, followed by magnitudes of about $[0.06 \ldots 0.3]$ for $T_{J M}, T_{1(1,0)}^{\operatorname{lin}}$ and $T_{2(1,0)}^{\operatorname{lin}} . "$

- p. 18, right, replace magnitudes: $0.3 \rightarrow 0.7,0.15 \rightarrow 0.3,0.05 \rightarrow 0.08$.

- p. 24, left, line $1:$ replace $0.3 \rightarrow 0.7$.

The relative sensitivities to variations of the polarisabilities remain unchanged. The corresponding Mathematica notebook, available from hgrie@gwu.edu, and the arXiv version (arXiv:1304.6594 [nucl-th]) have been adjusted accordingly, including new figures and corrections of a few inconsequential typographical errors.

I am very grateful to D.R. Phillips and R.W. Stewart whose persistence was instrumental in finding this error. This work was supported in part by the US-Department of Energy under contract DE-SC0015393, and by the Dean's Research Chair programme of the Columbian College of Arts and Sciences of The George Washington University.

\footnotetext{
a e-mail: hgrie@gwu.edu
} 